\title{
A BRCA1 deficient, NFkB driven immune signal predicts good outcome in triple negative breast cancer
}

\author{
Niamh E. Buckley ${ }^{1}$, Paula Haddock ${ }^{1}$, Ricardo De Matos Simoes ${ }^{1}$, Eileen Parkes ${ }^{1}$, \\ Gareth Irwin $^{1}$, Frank Emmert-Streib ${ }^{1}$, Stephen McQuaid ${ }^{1}$, Richard Kennedy ${ }^{1}$ and \\ Paul Mullan ${ }^{1}$ \\ ${ }^{1}$ Centre for Cancer Research and Cell Biology, Queen's University Belfast, Belfast, UK \\ Correspondence to: Niamh E. Buckley, email: n.obrien@qub.ac.uk \\ Keywords: triple negative breast cancer; BRCA1; NFkB; predictive biomarker; microenvironment \\ Received: February 11,2016 Accepted: February 18, $2016 \quad$ Published: March 02, 2016
}

\section{ABSTRACT}

Triple negative (TNBCs) and the closely related Basal-like (BLBCs) breast cancers are a loosely defined collection of cancers with poor clinical outcomes. Both show strong similarities with BRCA1-mutant breast cancers and BRCA1 dysfunction, or 'BRCAness', is observed in a large proportion of sporadic BLBCs. BRCA1 expression and function has been shown in vitro to modulate responses to radiation and chemotherapy. Exploitation of this knowledge in the treatment of BRCA1-mutant patients has had varying degrees of success. This reflects the significant problem of accurately detecting those patients with BRCA1 dysfunction. Moreover, not all BRCA1 mutations/loss of function result in the same histology/pathology or indeed have similar effects in modulating therapeutic responses. Given the poor clinical outcomes and lack of targeted therapy for these subtypes, a better understanding of the biology underlying these diseases is required in order to develop novel therapeutic strategies.

We have discovered a consistent NFKB hyperactivity associated with BRCA1 dysfunction as a consequence of increased Reactive Oxygen Species (ROS). This biology is found in a subset of BRCA1-mutant and triple negative breast cancer cases and confers good outcome. The increased NFKB signalling results in an anti-tumour microenvironment which may allow CD8+ cytotoxic $T$ cells to suppress tumour progression. However, tumours lacking this NFKB-driven biology have a more tumourpromoting environment and so are associated with poorer prognosis. Tumour-derived gene expression data and cell line models imply that these tumours may benefit from alternative treatment strategies such as reprogramming the microenvironment and targeting the IGF and AR signalling pathways.

\section{INTRODUCTION}

Breast cancer is a heterogeneous disease comprising of multiple tumour types that require different treatment approaches and have varied patient outcomes. Patient stratification, based on expression of the estrogen $(\mathrm{ER} \alpha)$ or Her2/neu/ERBB2 (Her2) receptors, has allowed for the use of targeted therapies such as Tamoxifen and Trastuzamab, respectively. Breast cancers that do not express these receptors are termed "triple negative breast cancers" (TNBCs) and have the poorest clinical outcome, reflecting the fact that they lack targeted therapies. All TNBCs are currently treated with DNA-damaging chemotherapy regimes such as FEC (5-FU, Epirubucin and Cyclophosphamide). They are a poorly defined subgroup with a large degree of heterogeneity suggesting that optimal treatment may only be attained by use of different treatment regimens. Gene expression microarray analyses of tumours has allowed breast cancers to be re-classified. These include the ER $\alpha$ and Her2 positive subgroups in addition to a Basal-like (BLBC) subgroup which is associated with the poorest clinical outcomes [1]. There is a high degree of overlap between the TNBC and BLBC subgroups with up to $70 \%$ of TNBCs displaying BLBC gene profiles as well as $77 \%$ of BLBCs classified as TNBC [2]. 
Hereditary breast cancers arising from mutation of the tumour suppressor gene, BRCA1, closely resemble sporadic TNBC/BLBCs while BRCA1 expression is downregulated in up to $30 \%$ of sporadic BLBCs [3]. BRCA1 function can therefore be abrogated both by mutation and downregulation of expression ('BRCAness') leading to tumourigenesis [4]. BRCA1 expression and function has been shown in vitro to modulate responses to radiation and chemotherapy[5, 6]. Exploitation of this knowledge in the treatment of BRCA1-mutant patients has had varying degrees of success $[7,8]$. This reflects the significant problem of accurately detecting those patients with BRCA1 dysfunction. Moreover, not all BRCA1 mutations/loss of function result in the same histology/pathology or indeed have similar effects in modulating therapeutic responses $[9,10]$. Therefore, an increased understanding of how discrete modes of BRCA1 dysfunction modulate disease biology and therapeutic responses is required.

BRCA1 tumours often display high numbers of infiltrating lymphocytes both intratumoural and in the surrounding stroma [11]. Moderate to extensive lymphocytic infiltrate (LI) is also observed in about half of all TNBC cases [12] and this is associated with good clinical outcome [12-14]. Indeed, a number of gene signatures based on activation of immune signalling have been developed in TNBCs, which predict for good outcome to current standard of care chemotherapy [1519]. Furthermore, it has been shown that BRCA1 mutant tumours tend to overexpress immune response genes [17, 20]. Whilst these studies suggest a role for the immune system in modulating TNBC responses, they do not elucidate the biology underlying the up-regulation of the immune response genes and the functional significance of the genes themselves in tumourigenesis.

The NFkB pathway primarily mediates the cellular response to external stimuli and plays a crucial role in regulating the immune response. Activation of the $\mathrm{NF} \kappa \mathrm{B}$ pathway underpins many aspects of cancer including survival, invasion and metastasis. BRCA1 has been shown to interact with p65 and acts as a transcriptional coactivator in response stimuli [21] and NFKB acts a critical mediator of BRCA1-induced chemoresistance [22]. However, in this study, we demonstrate that in the absence of functional BRCA1, basal NFKB activity is increased and NFKB target genes are increased in TNBC cell lines. A BRCA1 deficient, NFאB driven immune signal has been identified and this predicts good clinical outcome in TNBCs. This is underpinned by a favourable "M1-type" macrophage tumour microenvironment promoting active cytotoxic CD8+ infiltrate.

\section{RESULTS}

Using a NFאB luciferase reporter assay, higher NFKB activity is observed in BRCA1 mutant HCC1937
(Figure 1A(i)) and BRCA1 low MDA-MB-468 (Figure 1A(ii)) cells compared to their isogenic matched BRCA1 reconstituted controls. Conversely, shRNA mediated BRCA1 knockdown in the 184A1 normal breast cell line results in increased NFאB activity ((Figure 1A(iii)). Increased expression of known $\mathrm{NF} \kappa \mathrm{B}$ target genes was also observed in the absence of functional BRCA1 expression (Figure 1B(i-iii)). SiRNA against the p65 subunit of $\mathrm{NF} \kappa \mathrm{B}$ was used to demonstrate the increased expression in the absence of functional BRCA1 is dependent on the increased $\mathrm{NF} \kappa \mathrm{B}$ activity using CXCL1 as an exemplar NFkB-dependent gene (Figure 1B(iv)).

In order to delineate how loss of BRCA1 function results in increased basal NFkB activity, we used a series of inhibitors to pathways known to be regulated in a BRCA1-dependent fashion that can impact on NFאB activity such as Notch [23], DNA Damage Response (ATM and Parp inhibitors) [24, 25] and Reactive Oxygen Species (ROS) [26] (Figure 2A). Inhibition of ROS using NAC consistently resulted in a loss of increased NFKB activity observed in the absence of functional BRCA1. Consistent with this observation, ROS levels were significantly higher in cells lacking functional BRCA1 compared to their BRCA1 proficient controls (Figure 2B).

We next wanted to determine whether this observed biology was also present in breast cancer tumours. In order to achieve this, we created a cell line derived gene list of target genes specifically upregulated by $\mathrm{NF \kappa B}$ in the absence of functional BRCA1. Microarray analysis was carried out on BRCA1 mutant and reconstituted cells (HCC EV and BR) cells with and without siRNA targeted against the p65 subunit of NFאB (Supp Figure 2A). This list was refined to the smallest gene list with the most robust and significant fold changes (Supp Figure 2B). This gene list was then used to interrogate a TNBC microarray data set enriched for BRCA1 mutations [27] in order to identify a molecular subgroup of breast cancers enriched

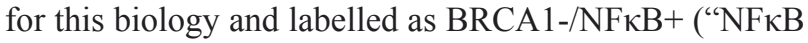
on") and all other tumours labelled as non-BRCA1-/

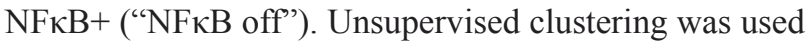
to take into account that not all BRCA1 mutations result in the same dysfunction [10] and that not all BRCA1 wildtype tumours possess functional BRCA1 [4] (Supp Figure 2B(ii)). In order to develop a tumour derived classifier gene signature to identify the BRCA1-/NFאB+ subgroup, an ElasticNet computational analysis was applied (Supp Figure 2B(iii) and Supp Table 1) and further refined based on most statistically significant fold changes (Supp Figure 2B(iv) and Supp Table 2) comprising of 42 genes most of which (39/42) are upregulated in the "NFאB on" subgroup. While this only contained one of our original cell line defined BRCA1/ NFkB target genes (CXCL10) this is not overly surprising as this is a tumour derived classifier. However, most of the cell line derived BRCA1/ NF $\kappa$ B genes were present in the differential gene list (DEG). Unfortunately, no clinical follow-up data was 
available for this cohort. Therefore, in order to determine the clinical significance of this "NFאB on" subgroup, the ElasticNet derived gene signature was applied to 4 additional TNBC datasets with available clinical follow up [18, 27-29]. Semi-supervised clustering, using the 42 gene

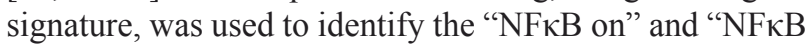
off" subgroups (Supp. Figure 3) and relapse free survival analysed (Figure 3). As shown in the Kaplan Meier curves, the "NFкB on" subgroup has significantly better relapse free survival. Cox Proportional Hazard analysis (Table 1) shows the "NFkB on" subgroups were 2.5 - 5 times less likely to relapse. Similar results were also observed for overall survival where data was available (Supp Figure 4). Multivariate analysis on the in-house TNBC cohort showed that the NFאB signature was independent of age, tumour size, chemotherapy regime and lymphovascular invasion (LVI) status but not lymph node involvement (Supp Table 3). Consistent with our in-vitro data, the "NFкB on" subgroup also expressed higher levels of genes associated with high ROS levels compared to the "NFkB off" subgroup (Supp Figure 5).

Given that the majority of the genes within the ElasticNet derived genelist are involved in immune response and the previously discussed high levels of immune cell infiltrate observed in BRCA1-mutant and TNBC, the next obvious step was to investigate whether the tumour microenvironment of these tumours

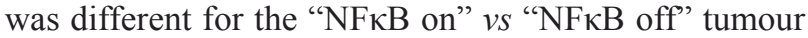

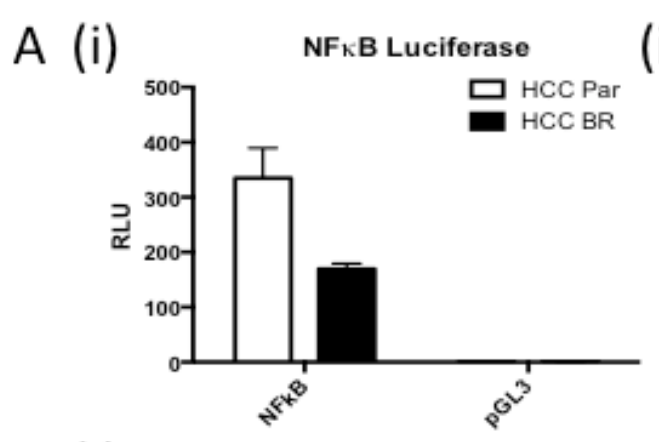

B (i)

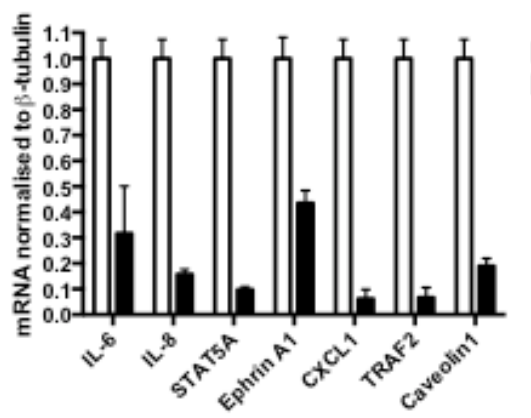

(iii)

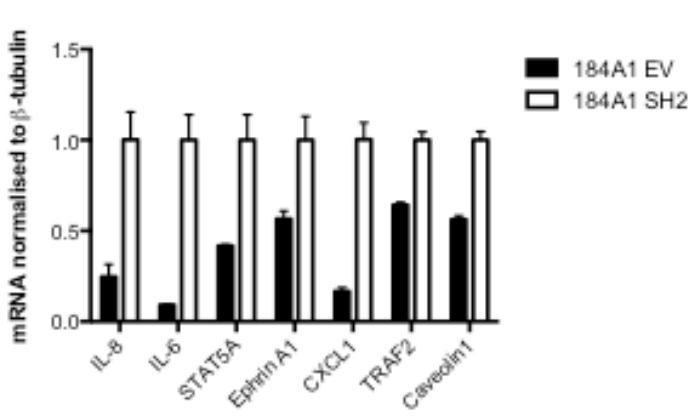

NFKB Luciferase
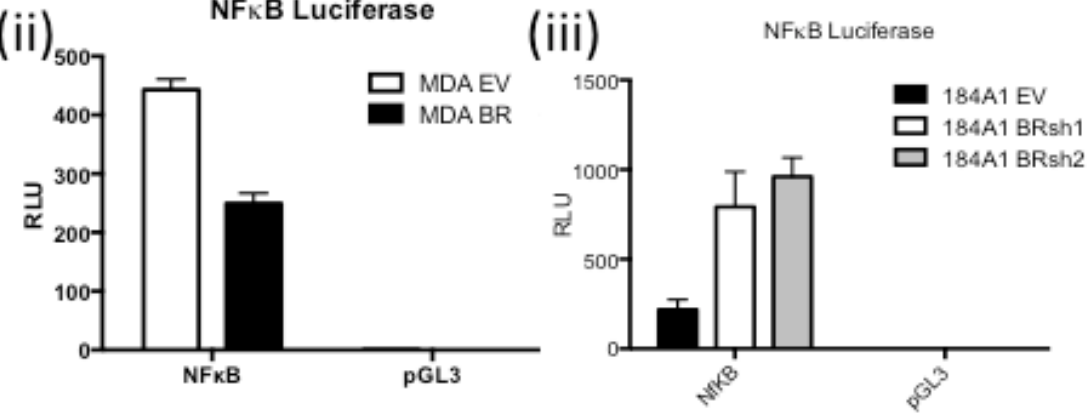

(ii)

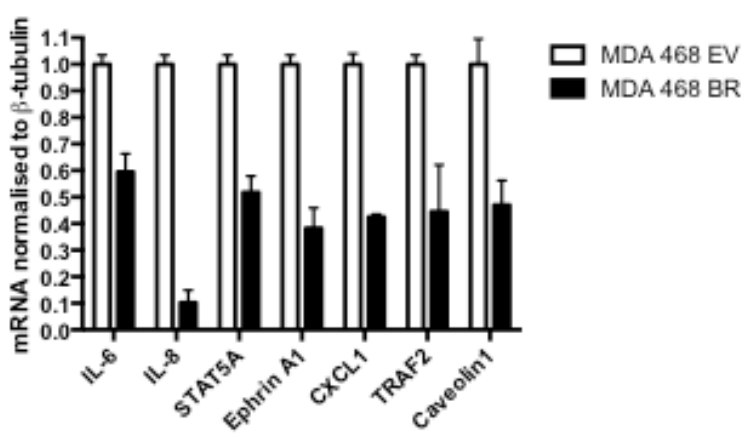

(iv)

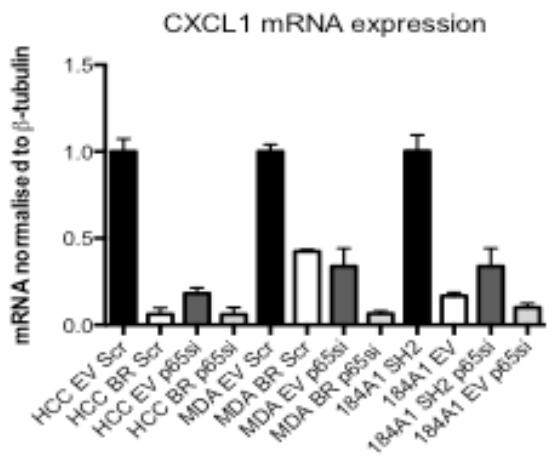

Figure 1: A. NFאB Luciferase Activity Assay of (i) HCC1937 (BRCA1 mutant) cells stably transfected with either empty vector (HCC EV) or full length BRCA1 (HCC BR), (ii) MDA468 (BRCA1 low) cells stably transfected with either empty vector (MDA EV) or full length BRCA1 (MDA BR), or (iii) 184A1 (normal breast) cells stably transfected with empty vector (EV) or BRCA1 shRNA (BRsh2). Cells were transfected with either NFkB reporter construct (NFkB) or the empty vector control (pGL3). Renilla was used to normalise for transfection efficiency. Values are expressed as relative luciferase units (RLU) normalised to pGL3 and Renilla. B. Real time PCR of NFאB target genes in (i) HCC EV and BR, (ii) MDA EV and BR and (iii) 184A1 EV and SH2 cells. $\beta$-tubulin was used as a housekeeper. Expression was then normalised to HCC EV, MDA EV and 184A1 SH2 respecively (iv) Real time PCR analysis of CXCL1 mRNA in HCC EV and BR, MDA EV and BR and 184A1 EV and SH2 cells transiently transfected with either scrambled control (scr) or p65 specific (p65si) siRNA for $72 \mathrm{hrs}$. $\beta$-tubulin was used as a housekeeper. 
A (i)

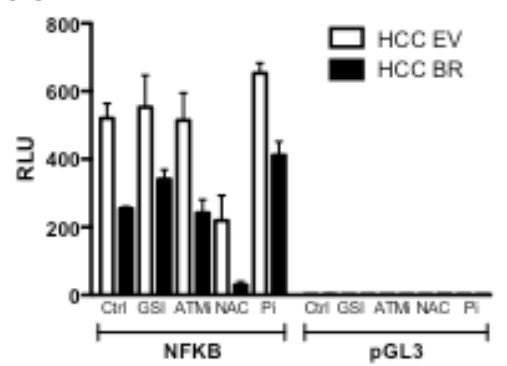

B (i)

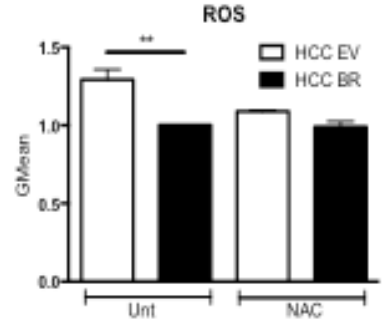

(ii)

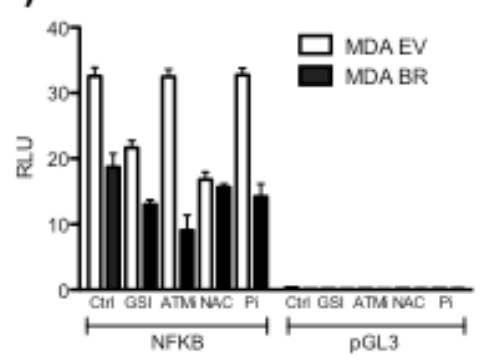

(ii)

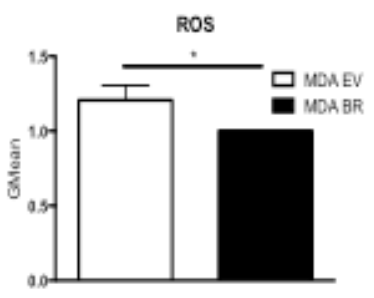

(iii)

NFkB Luciferase

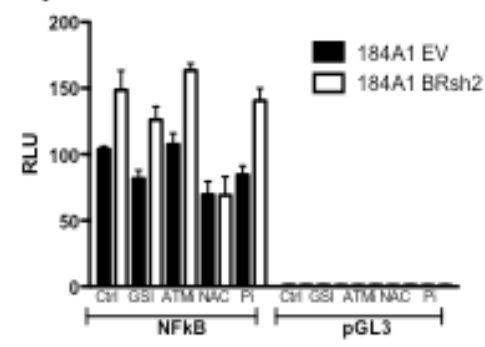

(iii)

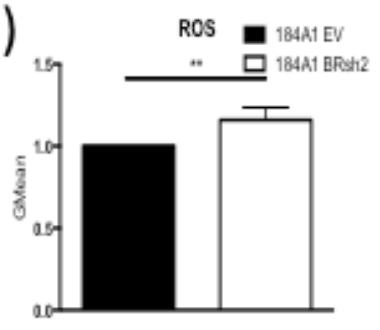

Figure 2: A. NFкB Luciferase Activity Assay of i) HCC EV and BR, (ii) MDA EV and BR and (iii) 184A1 EV and SH2 cells pre-treated for one hour with vehicle control (Ctrl), 250nM Gamma Secretase Inhibitor (GSI), 3.3 $\mu$ M ATM inhibitor (ATMi), $10 \mu \mathrm{M}$ NAC or $10 \mu \mathrm{M}$ Parp inhibitor $(\mathrm{Pi})$. Cells were then transfected with either NFkB reporter construct (NFkB) or the empty vector control (pGL3) with the relevant treatment. Renilla was used to normalise for transfection efficiency. Values are expressed as relative luciferase units (RLU) normalised to pGL3 and Renilla. B. Flow cytometry based analysis of Reactive Oxygen Species (ROS) using Carboxy-H2DCFDA in (i) HCC EV and BR cells treated with or without $10 \mu \mathrm{M}$ N-acetyl-L-cysteine (NAC), (ii) MDA EV and BR and (iii) 184A1 EV and SH2 cells.

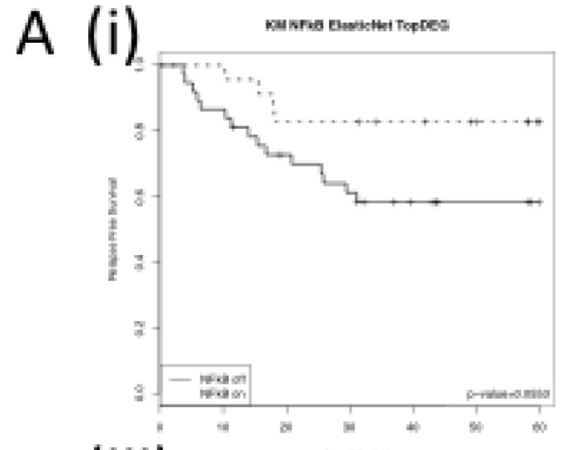

(iii)

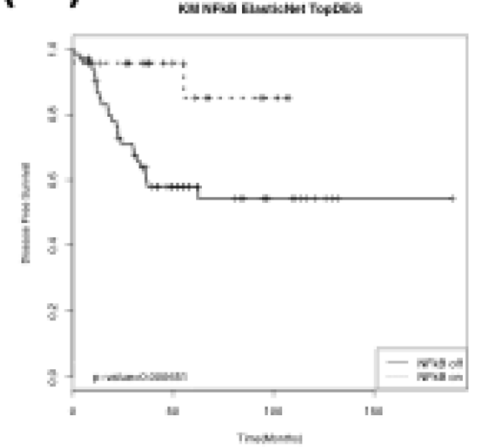

(ii)

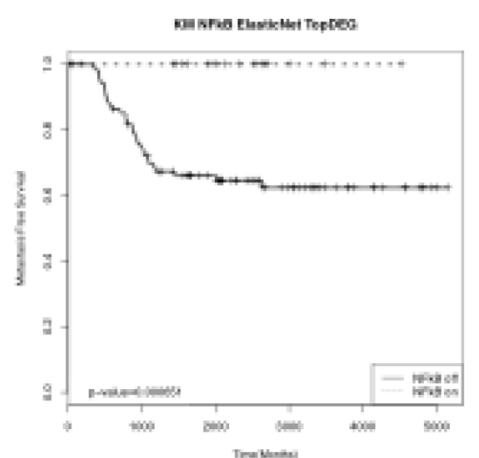

(iv)

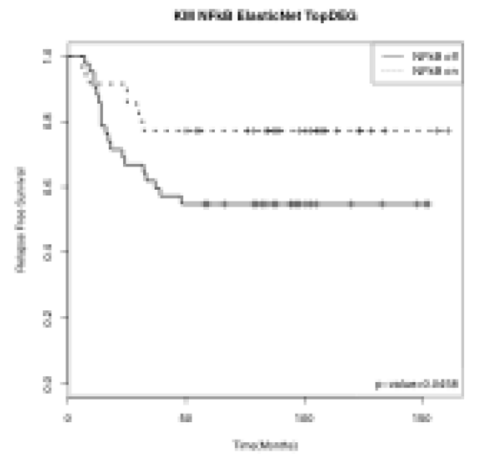

Figure 3: Kaplan Meier Curves of (i) the in- house Triple negative dataset and publically available (ii) GSE58812, (iii) GSE21653 and (iii) GSE2034 datasets stratified using the identified BRCA1-/NFkB+ (NFkB on) and non-BRCA1-/NFkB+ (NFkB off) groups. Log-rank p-values are shown. 
A (i)

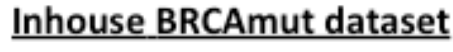

M2/M1 GES

(iv)

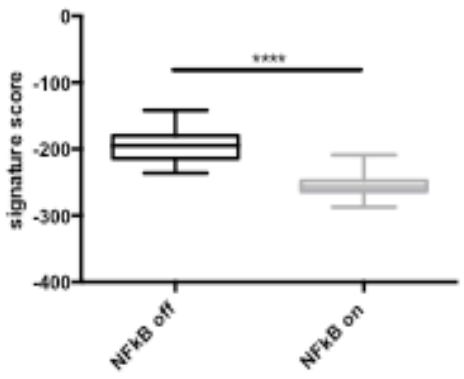

GSE21653 Sabatier

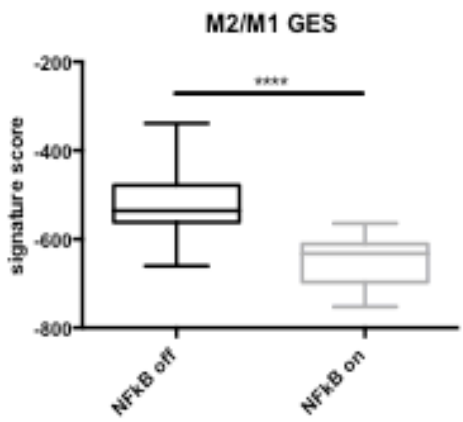

B (i)

Inhouse BRCAmut dataset

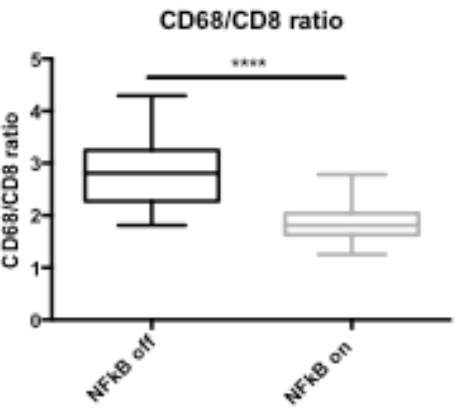

(iv)

GSE21653 Sabatier

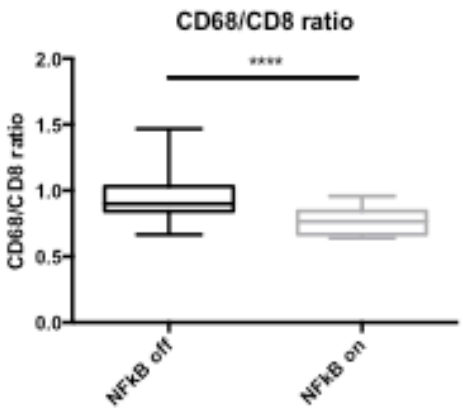

(ii)

Inhouse TNBC

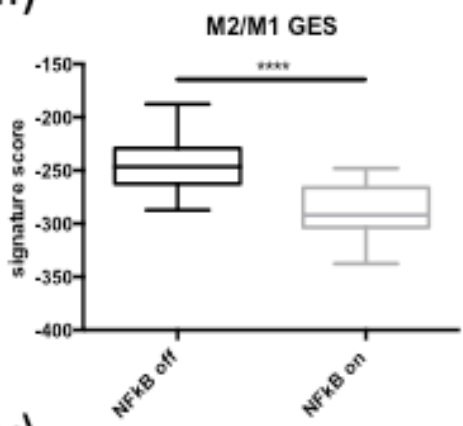

(v)

GSE2034 Wang

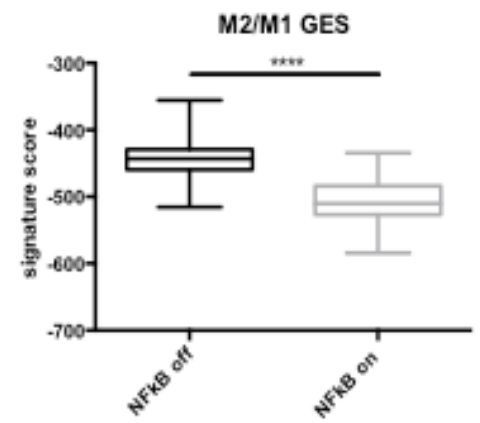

(ii)

Inhouse TNBC

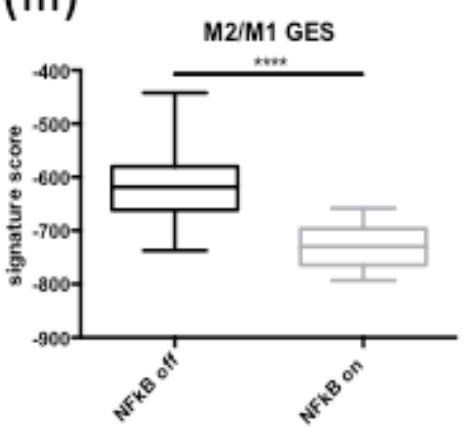

(iii) GS58812 Jezequel

(v)

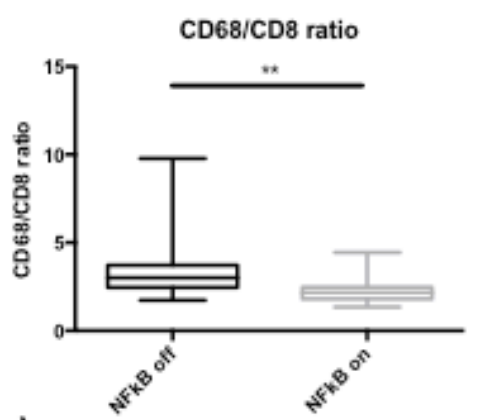

GSE2034 Wang

CD68/CD8 ratio

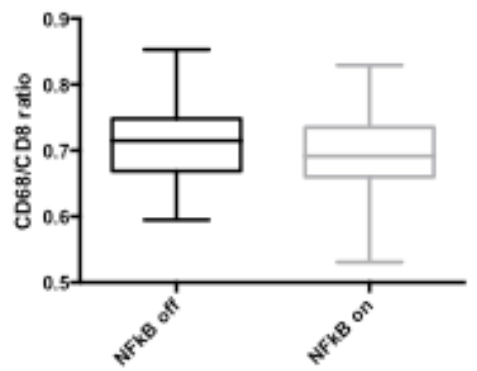

(iii) GSE58812 Jezequel

(iii) GSE58812 Jezequel
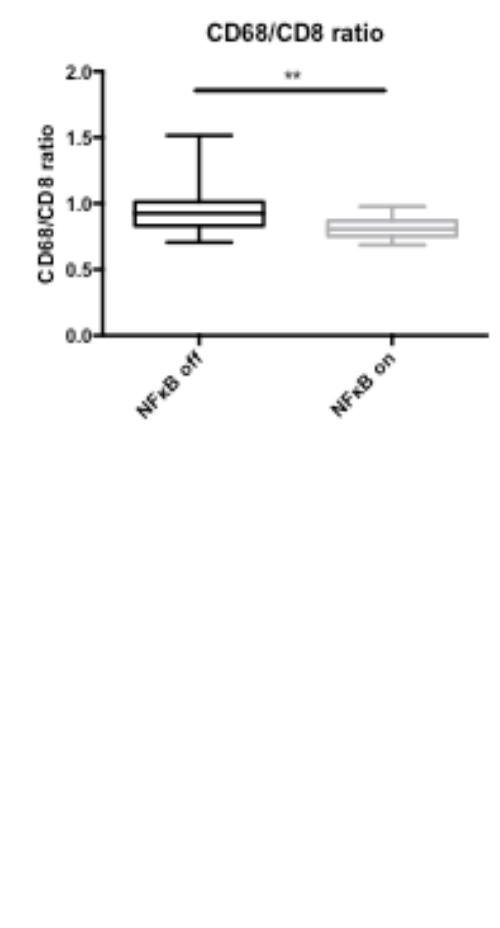

Figure 4: A. Box and Whisker plots of microarray derived M2/M1 Gene Expression Signature scores in (i) in house BRCA1 mutant dataset, (ii) in house TNBC dataset and publically available (iii) GSE58812, (iv) GSE21653 and (v) GSE2034 datasets. B. Box and Whisker plots of microarray derived CD68/CD8 expression ratios in (i) in house BRCA1 mutant dataset, (ii) in house TNBC dataset and publically available (iii) GSE58812, (iv) GSE21653 and (v) GSE2034 datasets. 
Table 1: Univariate Cox Proportional Hazard Ratio analysis of survival in the in-house TNBC and the publically available GSE58812, GSE21653 and GSE2034 data sets

\begin{tabular}{|l|l|l|l|l|l|}
\hline Relapse Free Survival & \multicolumn{3}{l|}{ Cox PH - Univariate } \\
\hline & & & HR & \%95 CI & $p$-value \\
\hline $\begin{array}{l}\text { Inhouse } \\
\text { TNBC }\end{array}$ & & $\begin{array}{l}\text { N(n) } \\
(19)\end{array}$ & & & \\
\hline $\begin{array}{l}\text { BRCA1/ } \\
\text { NFkB }\end{array}$ & off & $37(15)$ & 1 & & \\
\hline & on & $23(4)$ & 0.3559 & $\begin{array}{l}0.118- \\
1.073\end{array}$ & 0.0666 \\
\hline $\begin{array}{l}\text { GSE58812 } \\
\text { NFkB }\end{array}$ & off & $\begin{array}{l}\text { N(n) } \\
107(31)\end{array}$ & & & \\
\hline & on & $17(0)$ & $0.2886 *$ & $\begin{array}{l}0.1179- \\
0.7065\end{array}$ & 0.0065 \\
\hline $\begin{array}{l}\text { GSE21653 } \\
\text { NRCA1/ }\end{array}$ & off & $62(25)$ & 1 & & \\
\hline $\begin{array}{l}\text { NFkB } \\
\text { NRCA } 85\end{array}$ & on & $23(2)$ & 0.1956 & $\begin{array}{l}0.04632- \\
0.8263\end{array}$ & 0.0264 \\
\hline & on & $35(8)$ & 0.4412 & $\begin{array}{l}0.1929- \\
1.009\end{array}$ & 0.0525 \\
\hline $\begin{array}{l}\text { BRCA1/ } \\
\text { NFkB }\end{array}$ & off & $42(19)$ & 1 & & \\
\hline
\end{tabular}

* Cox PH HR not possible as no events in NFkB on, Mantel-Haenszel HR shown

Table 2: p-values of Chi-Squared and/or Fisher's exact tests of IHC-based correlations between "NFkB on" or "NFkB off" subgroups and immune markers

\begin{tabular}{|l|l|l|l|}
\hline \multicolumn{2}{|c|}{} & Chi Squared Test & Fisher's Exact Test \\
\hline \multirow{2}{*}{ N=53 } & \multicolumn{1}{|c|}{$\boldsymbol{p}$-value } & 0.0101 \\
\hline \multirow{2}{*}{ CD8 } & Intratumour & 0.0133 & - \\
\cline { 2 - 4 } & Stroma & 0.1124 & 0.0817 \\
\hline \multirow{2}{*}{ CD4 } & Intratumour & 0.0237 & - \\
\cline { 2 - 4 } & Stroma & 0.4129 & - \\
\hline \multirow{2}{*}{ FOXP3 } & Intratumour & 0.5061 & - \\
\cline { 2 - 4 } & Stroma & 0.4587 & - \\
\hline \multirow{2}{*}{ CD68 } & Intratumour & 0.2120 & - \\
\cline { 2 - 4 } & Stroma & 0.3290 & 0.0612 \\
\hline CD14 & & - & \\
\hline CD163 & & 0.0428 & \\
\hline
\end{tabular}

sub groups. Therefore, a TMA matched to the in-house triple negative breast cancer cohort was utilised. AntiCD8, -CD4 and -FOXP3 antibodies were used to assess cytotoxic, helper and regulatory T-cells, respectively. A separate score was given for T-cells located in the stroma $v s$ those within the tumour nest (intratumour). No significant differences in stromal CD4, CD8 or both stromal and intratumoural FOXP3 were observed. However, there was a significant correlation of CD8 and CD4 positive T cells within the tumour nest and the "NFкB on" subgroup (Table 2 and Supp Figure 6). Numerous studies have shown that the polarisation state of the tumour-associated macrophages can strongly influence a tumour-promoting or -destroying microenvironment [30, 31]. Simply, M1-like macrophages promote a $T_{H} 1$ response and tumour destruction while the M2-like macrophages promote a $\mathrm{T}_{\mathrm{H}} 2, \mathrm{CD} 8+$ suppressive, tumour promoting response. Given the fact that a number of the genes upregulated in the "NFאB on" subgroup are associated with interferon [32](Supp Figure 7) and the $\mathrm{T}_{\mathrm{H}} 1$ response (e.g. 
CXCL9, CXCL10), we postulated that the "NFאB on" subgroup may be associated with an M1-like macrophage environment. In order to test our hypothesis, we applied the M2/M1 gene expression signature (GES) developed by Jézéquel et al [18]. A highly significant correlation $(\mathrm{p} \leq 0.0001)$ was observed between a low M2/M1 score
A (i)

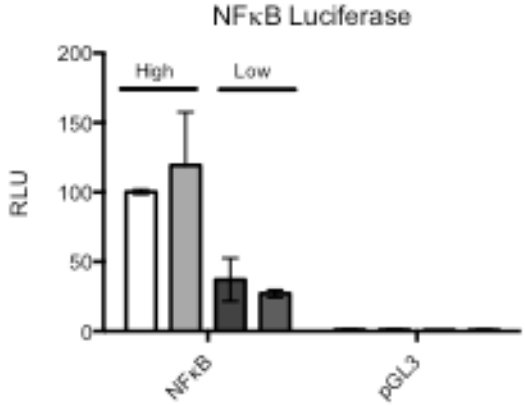

B (i)

(iv)
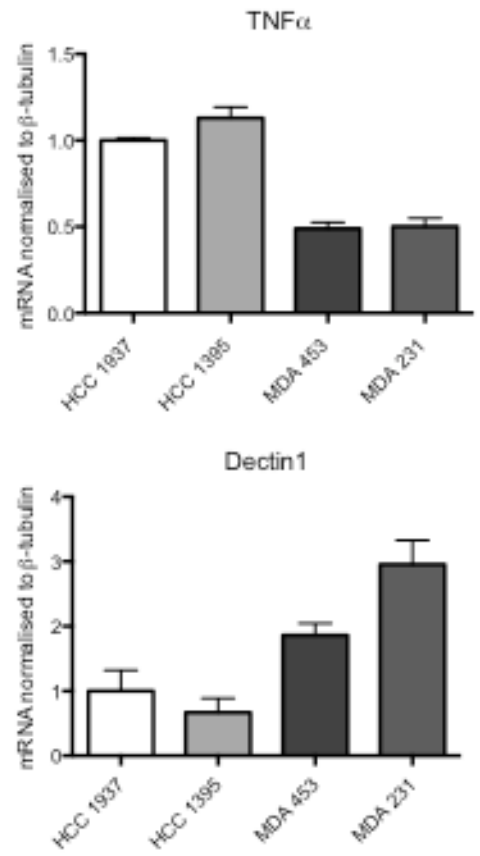

C (i)

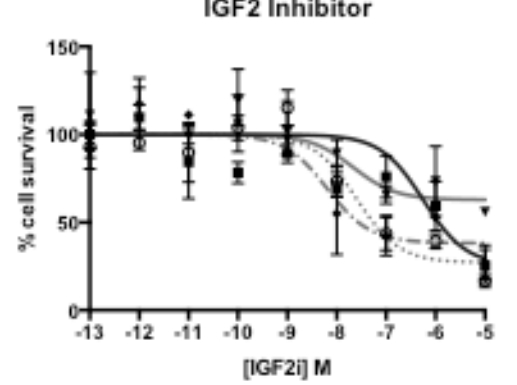

(ii)

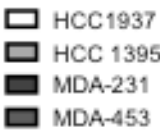

(ii)
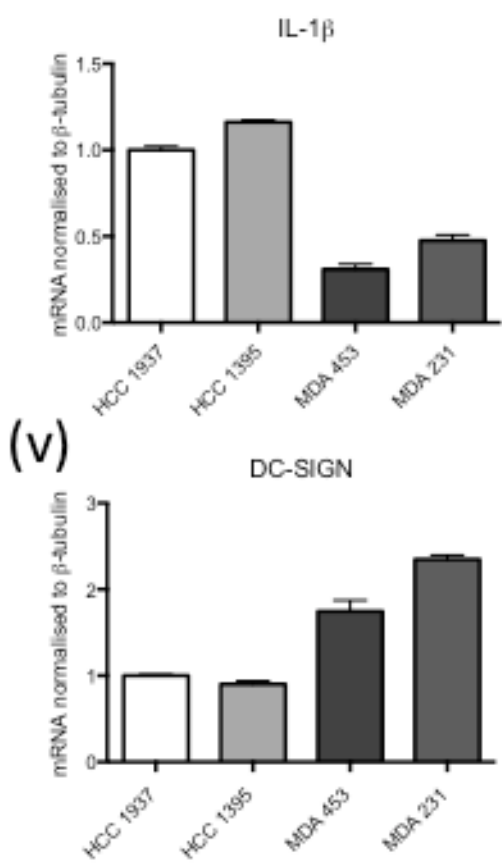

(ii)

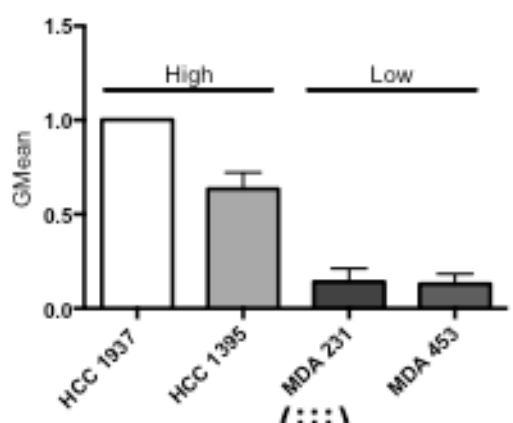

(iii)

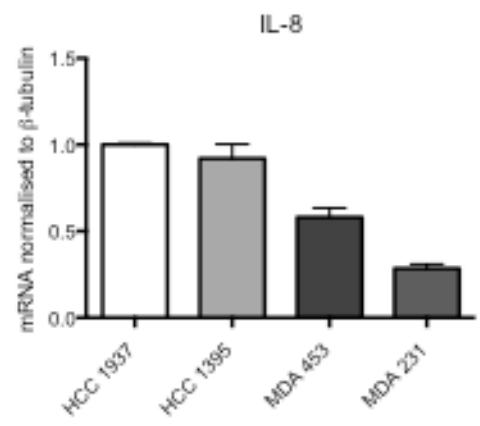

CDX
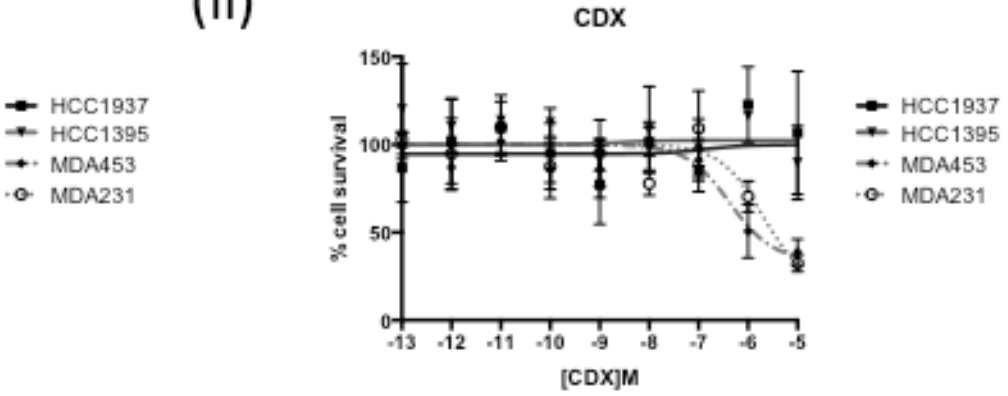

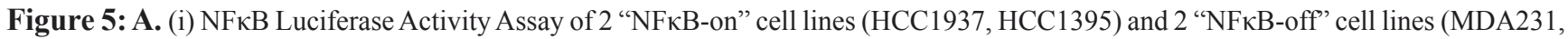
MDA453). Cells were transfected with either NFkB reporter construct (NFkB) or the empty vector control (pGL3). Renilla was used to normalise for transfection efficiency. Values are expressed as relative luciferase units (RLU) normalised to pGL3 and Renilla. (ii) Flow cytometry based analysis of Reactive Oxygen Species (ROS) using Carboxy-H2DCFDA in the same cell lines as (i). B. Real time PCR analysis of M1 and M2 macrophage markers in THP-1 cells co-cultured with media from "NFkB on" (HCC1937, HCC1395) and "NFkB off" (MDA231, MDA453) cells for 24 hours. $\beta$-tubulin was used as a housekeeper. C. Dose response curve of "NFkB on" (HCC1937, HCC1395) and "NFkB off"(MDA231, MDA453) treated with (i) IGF2 inhibitor or (ii) Bicalutamide. Cells were treated with the indicated range of concentration of drug for $72 \mathrm{hrs}$ before cell viability was assessed by MTT. Cell survival was normalised to vehicle control (100\%). 
(implying M1-like phenotype) and the "NFאB on" subgroup (Figure 4A). Alternatively, the CD68:CD8 ratio can also be analysed through microarray analysis and this has been shown to predict survival and chemotherapeutic response in breast cancer [33]. A high CD68:CD8 ratio implies a macrophage population suppressing the CD8 cytotoxic T-cells (e.g. M2-like) while a low score implies CD8+ T-cells mediated tumour suppression (e.g. M1-like). A significant correlation with a low CD68:CD8 ratio and the "NFkB on" subgroup was observed (Figure 4B). In order to validate the microarray-based gene expression data, the macrophage populations present in the TNBC TMA were assessed by IHC using the pan-macrophage marker, CD68. Consistent with the mRNA data, a higher CD68:CD8 ratio was observed in the "NFאB off" group compared to the "NFאB on" subgroup (Table 2 and Supp Figure 6). To assess M1/M2 polarisation, the CD14 and CD163 were also assessed by IHC [34, 35]. In keeping with the microarray-based data, higher CD14 staining was associated with "NFאB on" while higher CD163 was associated with the "NFkB off" subgroup (Table 2 and Supp Figure 6).

The results to date imply that the "NFkB on" subgroup respond well to the standard of care (SoC) DNA damaging chemotherapy used to treat TNBC (FEC +/D). Therefore novel therapeutic strategies are required to improve outcome in the "NFאB off" subgroup. While isogenic cells lines are a crucial research tool to elucidate downstream events from modulation of a single genetic event, they can never fully recapitulate the changes that would be seen in vivo with selective pressure from the tumour microenvironment. Given the integral role of immune signalling in this study, we therefore wanted to identify representative cell lines that may more closely mimic the downstream consequences of loss of BRCA1 function and subsequent deregulation of basal $\mathrm{NF} \kappa \mathrm{B}$ in vivo. Using TNBC cell lines from within two publically available cell line datasets $[36,37]$ and semisupervised clustering using the ElasticNet derived gene list, representative "NFkB on" (HCC1937 and HCC1395) and "NFאB off" (MDA231 and MDA453) cell lines were identified (Supp Figure 8). Interestingly, not all BRCA1 mutant cell lines were defined as "NFאB on". This is in keeping with the assumption that not all BRCA1 mutations result in the same dysfunction [10] and our own data showing that not all BRCA1 mutant tumours are classified

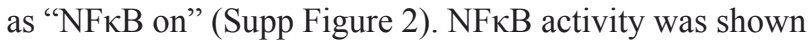

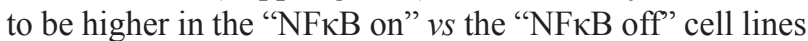
by luciferase activity assay (Figure 5A (i)) and consistent with the results in Figure 2, higher ROS levels were

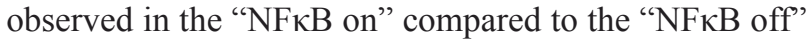
cell lines (Figure 5A (ii)). In order to demonstrate that the $\mathrm{NF} \kappa \mathrm{B}$ driven gene expression could regulate macrophage polarisation, tumour microenvironment and ultimately outcome in breast cancer cases, differentiated THP-1 cells were incubated with media from the four cell lines and then markers of M1 and M2 polarisation assessed by qPCR [38]. mRNA levels of the M1-associated markers (TNF- $\alpha$, IL-1 $\beta$ and IL-8) were all significantly higher in

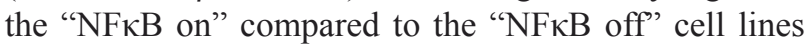
whilst the converse was seen with the M2-associated markers Dectin1 and DC-SIGN (Figure 5B). This implies that reprogramming of the microenvironment towards a more M1-like anti-tumour phenotype could be beneficial in the "NFкB off" subgroup. Furthermore, analysis of the ElasticNet derived genelist identified two "druggable" pathways up-regulated in the "NFKB off" compared to the "NFkB on" tumours namely the Androgen and IGF pathways. Microarray-based mRNA expression levels confirmed higher expression of IRS1, IGF2, IGF1R and

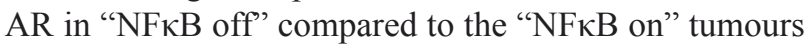
in all five datasets (Supp Figure 9). In order to assess the therapeutic implication of this, inhibitors against IGF2 and androgen receptor were tested in the four representative cell lines. "NFאB off" cell lines were significantly more sensitive to inhibition of these pathways than the "NFKB on" cell lines (Figure 5C).

\section{DISCUSSION}

In this study, we have shown that the absence of functional BRCA1, basal NFKB activity is increased due to increased ROS in vitro. This biology is found in a subset of BRCA1 mutant and TNBC cases and confers good outcome. The increased NFאB signalling results in an anti-tumour microenvironment which may allow CD8+ cytotoxic T cells to suppress tumour progression. However, tumours lacking this NFkB-driven biology have a more tumour-promoting environment and so are associated with poorer prognosis when treated with chemotherapy. Tumour-derived gene expression data and cell line models imply that these tumours may benefit from alternative treatment strategies such as reprogramming the microenvironment and targeting the IGF and AR signalling pathways.

This work highlights the fact that BRCA1-mutant and TNBCs are a heterogeneous groups of cancers that are not benefitting from the current "one size fits all" standard of care chemotherapy. This is further exemplified by use of PARP inhibitors to exploit the DNA repair defect of BRCA1 and BRCA2 mutant breast cancers. Despite good response rates in early clinical trials, no survival benefit has been demonstrated in BRCA1 mutant or TNBCs [8]. Therefore, biomarkers and potential therapeutic strategies must be developed around discreet biology downstream of specific modes of BRCA1-dysfunction. Indeed, a study by Fernandez-Ramies et al demonstrated that the immune response signature associated with ER-negative BRCA1 mutant tumours was modified by the type of BRCA1 mutation. BRCA1 mutant tumours harbouring truncating mutations (that probably led to a complete absence of protein through nonsense-mediated decay (NMD)) had 
a lower magnitude of expression of the immune genes compared to those harbouring missense mutations (resulting in an aberrant but still present BRCA1 protein) and this was underpinned by differing expression levels of the NF $\kappa$ B transcription factors [20]. Consistent with this, the majority of the tumours associated with that "NFKB on" subgroup harbour C-terminal mutations, which tend to avoid NMD and are expressed at relatively normal levels [39]. Furthermore, these mutations are commonly associated with loss of transcription regulation by BRCA1 [40] which is in keeping with our observations and known BRCA1-dependent regulation of $\mathrm{NF} \kappa \mathrm{B}$ function [21].

Our current study highlights the known variation in outcome of patients with TNBC where some patients do very poorly, relapse and die within the first 3 years while patients who do not recur within this period tend to have a much better prognosis [41]. Our data would suggest that the "NFкB on" subgroup respond well to the current standard of care DNA damaging chemotherapy (FEC +/-D) due in part to their favourable microenvironment. We would suggest however, that these patients probably would not benefit from the addition of docetaxel to their regime regardless of lymph-node involvement, given that tumours with dysfunctional BRCA1 are less likely to respond to anti-microtubule agents [42]. Counterintuitively, it is interesting to note higher gene expression of the PD1 ligand, PD-L1 (CD274) in this subgroup. This implies that these tumours may also benefit from an immune checkpoint blockade strategy. This emphasises the complexity of dialogue between the tumour cell and the cells within the microenvironment such as T-cells and macrophages [31]. We suggest that the NFאB-driven signal from within the tumour cells promotes a $\mathrm{M} 1 / \mathrm{T}_{\mathrm{H}} 1$ microenvironment that then produce their own signals to enhance and maintain this and influence responses to chemotherapy and overall outcome. Therefore, the "NFאB off" subgroup may benefit from a more taxanebased chemotherapeutic regime (FEC-D rather than FEC as first line treatment) or targeted therapies (alone or in combination) to re-program the microenvironment towards a more M1-like anti-tumour scenario. A number of these drugs have been developed (e.g. the CD-20 targeting agent, Rituximab) and show promise in in vivo models and in patients [30]. Furthermore, reprogramming of macrophages may be a more favourable approach compared to eradication, as they may be required for interaction with other components of the microenvironment [43]. In addition, our data suggests that use of therapies targeting the IGF and AR signalling pathway may also benefit this subgroup. These pathways have already been identified as tractable drug targets within TNBC with clinical trial results showing promise [44]. We believe that this NFאB-driven biology may allow stratification to predict who is likely to respond from such therapies.

In summary, we have identified a BRCA1-deficient,
NFkB-driven biology that predicts good outcome in TNBC due to the promotion of a favourable tumour microenvironment where immune targeting of the tumours is more efficient. Knowledge of this can be used to improve poorer outcome patients through macrophage reprogramming or use of specific targeted therapies.

\section{MATERIALS AND METHODS}

\section{Cell lines}

All cell lines were purchased from ATCC and maintained as directed except for the 184A1 cells which were a kind gift from Dr Martha Stampfer (University of California) and maintained as previously described [45]. Cell lines were characterised by isoenzyme/cytochrome c oxidase I (COI) assay and short tandem repeat (STR) analysis by the cell bank. Full details of the HCC-EV/ $\mathrm{BR}$ and MDA468-EV/BR cell lines are provided in [46]. 184A1-EV and -BRsh2 cells were generated by stable lentivirus transfection of the $184 \mathrm{~A} 1$ cells with pll3.7-EV or BRsh2 respectively (a kind gift from Prof. Wicha). Infected cells were selected in the presence of $1 \mu \mathrm{g} / \mu \mathrm{l}$ puromycin. For drug treatments, cells were treated with the relevant concentration of IGF2 inhibitor (Sigma, UK) or Bicalutamide (Sigma, UK) for $72 \mathrm{hrs}$ before cell viability was assessed by MTT (Sigma, UK).

\section{Short interfering RNA (siRNA)}

Transfections were done using RNAiMax reagent (Invitrogen, UK), as outlined in the manufacturer's instructions. siRNA oligonucleotide were obtained from Eurofins and used at a final concentration of 10nM.

\section{Western blot analysis}

Protein lysates were extracted in EDTA Lysis Buffer (ELB) $(0.25 \mathrm{M} \mathrm{NaCl}, 0.1 \%$ IEPGAL, $0.25 \mathrm{M}$ Hepes, 5mM EDTA, 0.5mM DTT), separated on a SDS PAGE gel, transferred to a PVDF membrane followed by immunoblotting. Antibodies are previously described in [23]

\section{RNA extraction, reverse transcription and real time quantitative PCR (RqPCR)}

RNA was extracted using RNA STAT60 Total RNA extraction Reagent (Tel-Test Inc, USA), reverse transcribed using the Transcriptor First Strand cDNA Synthesis kit (Roche, UK) and RqPCR analysis performed on the LC96 (Roche, UK) using Sybr Green (Roche, UK) 
according to the manufacturer's instructions. Primers used are listed in Supplementary Data and [38].

\section{Luciferase assays}

NFkB-pGL3 has been previously described [47]. Cells were co-transfected with the relevant Luciferase constructs and Renilla using GeneJuice (Novagen, Germany) according to the manufacturer's instructions. After 24hrs, cells were lysed with Passive Lysis Buffer (Promega, UK) and Luciferase and Renilla activity assessed by luminescence using D-Luciferin and Coelenterazine as substrates, respectively. For treatments with Gamma Secretase Inhibitor (Calbiochem, Germany), ATM inhibitor (KU60019 Tocris Biosciences, UK), NAC (Sigma, UK) and Parp Inhibitor (Olaparib, Selleckchem, UK), cells were pre-treated for $1 \mathrm{hr}$ prior to transfections with luciferase constructs and maintained in drug for the length of the experiment.

\section{Reactive oxygen species detection}

Cells were incubated with $5 \mu \mathrm{M}$ CM-H2DCFDA (Invitrogen, UK) for 30 minutes followed by flow cytometry. TeBOOH was included as a positive control in all experiments.

\section{Tissue microarrays (TMAs)}

The breast cancer TMAs used in this study were constructed from Formalin-fixed paraffin-embedded primary tumour blocks by the Northern Ireland Biobank and have been previously described [48]. Each tumour sample was represented by three independent cores. TNBC cases from within this cohort were identified from associated clinical and pathological information. Repeat IHC for ER, PR and HER2 on the TMA sections confirmed the TNBC status of this case cohort. Full details of antibodies used are listed in Supplementary Data. All antibodies were scored independently by two histopathologists blinded to patient clinicopathological and outcome data. Immune markers were scored on a 0-3 scoring system with representative images in Supplementary Figure 10.

\section{Microarrays}

Microarray analyses were performed as previously described on the Almac Breast DSA [49]. NFאB target genes were determined by identifying BRCA1 and p65 regulated genes independently (using the $\mathrm{R}$ package "limma") and then overlapping the gene lists. TNBC samples were identified (if required) from within the public datasets $[18,27-29]$ using the associated clinical information. BRCA2 mutant samples were excluded from discovery dataset [27].

\section{ElasticNet}

The ElasticNet regularization procedure was performed using the R package "glmnet" [50]. The optimal lambda was chosen based on a 10-fold cross-validation. The ElasticNet regularization is a convex combination of the ridge and the lasso penalty with a weighting parameter "alpha" (0.3 was used). Bootstrapping (x100) followed by a hypergeometric test to identify non-random features was used for feature selection.

\section{Survival analysis}

All Kaplan Meier Curves and Hazard Ratio Calculations were carried out using the $\mathrm{R}$ package "Survival".

\section{Statistical analysis}

All relevant data was analyzed by two-tailed Students $t$-test. All data was deemed significant with a $\mathrm{p}$-value of at least $<0.05$. All $p$-values are included in Supplementary Data.

\section{ACKNOWLEDGMENTS}

The samples used in this research were received from the Northern Ireland Biobank which is funded by HSC Research and Development Division of the Public Health Agency in Northern Ireland and Cancer Research UK through the Belfast CR-UK Centre and the Northern Ireland Experimental Cancer Medicine Centre; additional support was received from the Friends of the Cancer Centre. The Northern Ireland Molecular Pathology Laboratory, which is responsible for creating resources for the NIB, has received funding from Cancer Research UK, the Friends of the Cancer Centre and the Sean Crummey Foundation.

\section{CONFLICTS OF INTEREST}

The authors declare no conflict of interest

\section{GRANT SUPPORT}

This work was funded by Breast Cancer Now (May2012SF122). 


\section{Editorial note}

This paper has been accepted based in part on peerreview conducted by another journal and the authors' response and revisions as well as expedited peer-review in Oncotarget.

\section{REFERENCES}

1. Sorlie T, Perou CM, Tibshirani R, Aas T, Geisler S, Johnsen H, Hastie T, Eisen MB, van de Rijn M, Jeffrey SS, Thorsen T, Quist H, Matese JC, Brown PO, Botstein $\mathrm{D}$, Eystein Lonning $\mathrm{P}$, et al. Gene expression patterns of breast carcinomas distinguish tumor subclasses with clinical implications. PNAS. 2001; 98:10869-10874.

2. Bertucci F, Finetti P, Cervera N, Esterni B, Hermitte F, Viens $\mathrm{P}$ and Birnbaum D. How basal are triple-negative breast cancers? Int J Cancer. 2008; 123:236-240.

3. Yang Q, Sakurai T, Mori I, Yoshimura G, Nakamura M, Nakamura Y, Suzuma T, Tamaki T, Umemura T, Kakudo $\mathrm{K}$. Prognostic significance of BRCA1 expression in Japanese sporadic breast carcinomas. Cancer. 2001; 92:5460 .

4. Turner N, Tutt A and Ashworth A. Hallmarks of 'BRCAness' in sporadic cancers. Nat Rev Cancer. 2004; 4:814-819.

5. Abbott DW, Thompson ME, Robinson-Benion C, Tomlinson G, Jensen RA and Holt JT. BRCA1 expression restores radiation resistance in BRCA1-defective cancer cells through enhancement of transcription-coupled DNA repair. J Biol Chem. 1999; 274:18808-18812.

6. Kennedy RD, Quinn JE, Mullan PB, Johnston PG and Harkin DP. The role of BRCA1 in the cellular response to chemotherapy. Journal of the National Cancer Institute. 2004; 96:1659-1668.

7. James CR, Quinn JE, Mullan PB, Johnston PG and Harkin DP. BRCA1, a potential predictive biomarker in the treatment of breast cancer. Oncologist. 2007; 12:142-150.

8. O'Shaughnessy J, Schwartzberg L, Danso M, Rugo H, Miller K, Yardley D, Carlson R, Finn R, Charpentier E, Freese M, Gupta S, Blackwood-Chirchir A and Winer EP. A radomized phase III study of iniparib (BSI-201) in combination with gemcitabine/carboplatin $(\mathrm{G} / \mathrm{C})$ in metastatic triple negative breast cancer. Journal of Clinical Oncology. 2011; 29 (Supplement).

9. Morris JR, Pangon L, Boutell C, Katagiri T, Keep NH and Solomon E. Genetic analysis of BRCA1 ubiquitin ligase activity and its relationship to breast cancer susceptibility. Human molecular genetics. 2006; 15:599-606.

10. Linger RJ and Kruk PA. BRCA1 16 years later: riskassociated BRCA1 mutations and their functional implications. Febs J. 2010; 277:3086-3096.

11. Lakhani SR, Jacquemier J, Sloane JP, Gusterson BA, Anderson TJ, van de Vijver MJ, Farid LM, Venter D,
Antoniou A, Storfer-Isser A, Smyth E, Steel CM, Haites N, Scott RJ, Goldgar D, Neuhausen S, et al. Multifactorial analysis of differences between sporadic breast cancers and cancers involving BRCA1 and BRCA2 mutations. Journal of the National Cancer Institute. 1998; 90:1138-1145.

12. Kreike B, van Kouwenhove M, Horlings H, Weigelt B, Peterse H, Bartelink $\mathrm{H}$ and van de Vijver MJ. Gene expression profiling and histopathological characterization of triple-negative/basal-like breast carcinomas. Breast Cancer Res. 2007; 9:R65.

13. Liu S, Lachapelle J, Leung S, Gao D, Foulkes WD and Nielsen TO. CD8+ lymphocyte infiltration is an independent favorable prognostic indicator in basal-like breast cancer. Breast Cancer Res. 2012; 14:R48.

14. Baker K, Lachapelle J, Zlobec I, Bismar TA, Terracciano $\mathrm{L}$ and Foulkes WD. Prognostic significance of CD8+ T lymphocytes in breast cancer depends upon both oestrogen receptor status and histological grade. Histopathology. 2011; 58:1107-1116.

15. Yau C, Esserman L, Moore DH, Waldman F, Sninsky J and Benz CC. A multigene predictor of metastatic outcome in early stage hormone receptor-negative and triple-negative breast cancer. Breast Cancer Res. 2010; 12:R85.

16. Hanker LC, Rody A, Holtrich U, Pusztai L, Ruckhaeberle E, Liedtke C, Ahr A, Heinrich TM, Sanger N, Becker S and Karn T. Prognostic evaluation of the B cell/IL-8 metagene in different intrinsic breast cancer subtypes. Breast Cancer Res Treat. 2013; 137:407-416.

17. Teschendorff AE, Miremadi A, Pinder SE, Ellis IO and Caldas C. An immune response gene expression module identifies a good prognosis subtype in estrogen receptor negative breast cancer. Genome biology. 2007; 8:R157.

18. Jezequel P, Loussouarn D, Guerin-Charbonnel C, Campion L, Vanier A, Gouraud W, Lasla H, Guette C, Valo I, Verriele $\mathrm{V}$ and Campone M. Gene-expression molecular subtyping of triple-negative breast cancer tumours: importance of immune response. Breast Cancer Res. 2015; 17:43.

19. Rody A, Karn T, Liedtke C, Pusztai L, Ruckhaeberle E, Hanker L, Gaetje R, Solbach C, Ahr A, Metzler D, Schmidt M, Muller V, Holtrich U and Kaufmann M. A clinically relevant gene signature in triple negative and basal-like breast cancer. Breast Cancer Res. 2011; 13:R97.

20. Fernandez-Ramires R, Sole X, De Cecco L, Llort G, Cazorla A, Bonifaci N, Garcia MJ, Caldes T, Blanco I, Gariboldi M, Pierotti MA, Pujana MA, Benitez J and Osorio A. Gene expression profiling integrated into network modelling reveals heterogeneity in the mechanisms of BRCA1 tumorigenesis. British journal of cancer. 2009; 101:1469-1480.

21. Benezra M, Chevallier N, Morrison DJ, MacLachlan TK, El-Deiry WS and Licht JD. BRCA1 augments transcription by the NF-kappaB transcription factor by binding to the Rel domain of the p65/RelA subunit. J Biol Chem. 2003; 278:26333-26341. 
22. Harte MT, Gorski JJ, Savage KI, Purcell JW, Barros EM, Burn PM, McFarlane C, Mullan PB, Kennedy RD, Perkins ND and Harkin DP. NF-kappaB is a critical mediator of BRCA1-induced chemoresistance. Oncogene. 2014; 33:713-723.

23. Buckley NE, Nic An Tsaoir CB, Blayney JK, Oram LC, Crawford NT, D'Costa ZC, Quinn JE, Kennedy RD, Harkin DP and Mullan PB. BRCA1 is a key regulator of breast differentiation through activation of Notch signalling with implications for anti-endocrine treatment of breast cancers. Nucleic Acids Res. 2013; 41:8601-14. doi: 10.1093/nar/ gkt626.

24. Zerfaoui M, Errami Y, Naura AS, Suzuki Y, Kim H, Ju J, Liu T, Hans CP, Kim JG, Abd Elmageed ZY, Koochekpour S, Catling A and Boulares AH. Poly(ADP-ribose) polymerase-1 is a determining factor in Crm1-mediated nuclear export and retention of p65 NF-kappa B upon TLR4 stimulation. Journal of immunology. 2010; 185:1894-1902.

25. Wu ZH, Shi Y, Tibbetts RS and Miyamoto S. Molecular linkage between the kinase ATM and NF-kappaB signaling in response to genotoxic stimuli. Science. 2006; 311:11411146.

26. Gloire G, Legrand-Poels S and Piette J. NF-kappaB activation by reactive oxygen species: fifteen years later. Biochemical pharmacology. 2006; 72:1493-1505.

27. Mulligan JM, Hill LA, Deharo S, Irwin G, Boyle D, Keating KE, Raji OY, McDyer FA, O’Brien E, Bylesjo M, Quinn JE, Lindor NM, Mullan PB, James CR, Walker SM, Kerr $\mathrm{P}$, et al. Identification and validation of an anthracycline/ cyclophosphamide-based chemotherapy response assay in breast cancer. Journal of the National Cancer Institute. 2014; 106:djt335.

28. Sabatier R, Finetti P, Cervera N, Lambaudie E, Esterni B, Mamessier E, Tallet A, Chabannon C, Extra JM, Jacquemier J, Viens P, Birnbaum D and Bertucci F. A gene expression signature identifies two prognostic subgroups of basal breast cancer. Breast Cancer Res Treat. 2011; 126:407-420.

29. Wang Y, Klijn JG, Zhang Y, Sieuwerts AM, Look MP, Yang F, Talantov D, Timmermans M, Meijer-van Gelder ME, Yu J, Jatkoe T, Berns EM, Atkins D and Foekens JA. Gene-expression profiles to predict distant metastasis of lymph-node-negative primary breast cancer. Lancet. 2005; 365:671-679.

30. Ruffell B and Coussens LM. Macrophages and Therapeutic Resistance in Cancer. Cancer Cell. 2015; 27:462-472.

31. Biswas SK and Mantovani A. Macrophage plasticity and interaction with lymphocyte subsets: cancer as a paradigm. Nature immunology. 2010; 11:889-896.

32. Rusinova I, Forster S, Yu S, Kannan A, Masse M, Cumming $\mathrm{H}$, Chapman $\mathrm{R}$ and Hertzog PJ. Interferome v2.0: an updated database of annotated interferon-regulated genes. Nucleic Acids Res. 2013; 41:D1040-1046.

33. DeNardo DG, Brennan DJ, Rexhepaj E, Ruffell B, Shiao SL, Madden SF, Gallagher WM, Wadhwani N, Keil SD,
Junaid SA, Rugo HS, Hwang ES, Jirstrom K, West BL and Coussens LM. Leukocyte complexity predicts breast cancer survival and functionally regulates response to chemotherapy. Cancer discovery. 2011; 1:54-67.

34. van Dongen M, Savage ND, Jordanova ES, Briaire-de Bruijn IH, Walburg KV, Ottenhoff TH, Hogendoorn $\mathrm{PC}$, van der Burg SH, Gelderblom $\mathrm{H}$ and van Hall $\mathrm{T}$. Anti-inflammatory M2 type macrophages characterize metastasized and tyrosine kinase inhibitor-treated gastrointestinal stromal tumors. Int J Cancer. 2010; 127:899-909.

35. Yang J, Zhang L, Yu C, Yang XF and Wang H. Monocyte and macrophage differentiation: circulation inflammatory monocyte as biomarker for inflammatory diseases. Biomarker research. 2014; 2:1.

36. Neve RM, Chin K, Fridlyand J, Yeh J, Baehner FL, Fevr T, Clark L, Bayani N, Coppe JP, Tong F, Speed T, Spellman PT, DeVries S, Lapuk A, Wang NJ, Kuo WL, et al. A collection of breast cancer cell lines for the study of functionally distinct cancer subtypes. Cancer Cell. 2006; 10:515-527.

37. Garnett MJ, Edelman EJ, Heidorn SJ, Greenman CD, Dastur A, Lau KW, Greninger P, Thompson IR, Luo X, Soares J, Liu Q, Iorio F, Surdez D, Chen L, Milano RJ, Bignell GR, et al. Systematic identification of genomic markers of drug sensitivity in cancer cells. Nature. 2012; 483:570-575.

38. Chanput W, Mes JJ, Savelkoul HF and Wichers HJ. Characterization of polarized THP-1 macrophages and polarizing ability of LPS and food compounds. Food \& function. 2013; 4:266-276.

39. Perrin-Vidoz L, Sinilnikova OM, Stoppa-Lyonnet D, Lenoir GM and Mazoyer S. The nonsense-mediated mRNA decay pathway triggers degradation of most BRCA1 mRNAs bearing premature termination codons. Human molecular genetics. 2002; 11:2805-2814.

40. Mullan PB, Quinn JE and Harkin DP. The role of BRCA1 in transcriptional regulation and cell cycle control. Oncogene. 2006; 25:5854-5863.

41. Dent R, Trudeau M, Pritchard KI, Hanna WM, Kahn HK, Sawka CA, Lickley LA, Rawlinson E, Sun P and Narod SA. Triple-negative breast cancer: clinical features and patterns of recurrence. Clin Cancer Res. 2007; 13:4429-4434.

42. Quinn JE, Kennedy RD, Mullan PB, Gilmore PM, Carty M, Johnston PG and Harkin DP. BRCA1 functions as a differential modulator of chemotherapy-induced apoptosis. Cancer Res. 2003; 63:6221-6228.

43. Affara NI, Ruffell B, Medler TR, Gunderson AJ, Johansson M, Bornstein S, Bergsland E, Steinhoff M, Li Y, Gong Q, Ma Y, Wiesen JF, Wong MH, Kulesz-Martin M, Irving B and Coussens LM. B cells regulate macrophage phenotype and response to chemotherapy in squamous carcinomas. Cancer Cell. 2014; 25:809-821.

44. Gucalp A, Tolaney S, Isakoff SJ, Ingle JN, Liu MC, Carey LA, Blackwell K, Rugo H, Nabell L, Forero A, Stearns V, 
Doane AS, Danso M, Moynahan ME, Momen LF, Gonzalez JM, et al. Phase II trial of bicalutamide in patients with androgen receptor-positive, estrogen receptor-negative metastatic Breast Cancer. Clin Cancer Res. 2013; 19:55055512.

45. Garbe JC, Bhattacharya S, Merchant B, Bassett E, Swisshelm K, Feiler HS, Wyrobek AJ and Stampfer MR. Molecular distinctions between stasis and telomere attrition senescence barriers shown by long-term culture of normal human mammary epithelial cells. Cancer Res. 2009; 69:7557-7568.

46. Buckley NE, D'Costa Z, Kaminska M and Mullan PB. $\mathrm{S} 100 \mathrm{~A} 2$ is a BRCA1/p63 coregulated tumour suppressor gene with roles in the regulation of mutant p53 stability. Cell death \& disease. 2014; 5:e1070.

47. Wilson C, Purcell C, Seaton A, Oladipo O, Maxwell PJ, O'Sullivan JM, Wilson RH, Johnston PG and Waugh DJ. Chemotherapy-induced CXC-chemokine/CXC-chemokine receptor signaling in metastatic prostate cancer cells confers resistance to oxaliplatin through potentiation of nuclear factor-kappaB transcription and evasion of apoptosis. The Journal of pharmacology and experimental therapeutics. 2008; 327:746-759.
48. Boyle DP, McArt DG, Irwin G, Wilhelm-Benartzi CS, Lioe TF, Sebastian E, McQuaid S, Hamilton PW, James JA, Mullan PB, Catherwood MA, Harkin DP and Salto-Tellez $M$. The prognostic significance of the aberrant extremes of p53 immunophenotypes in breast cancer. Histopathology. 2014; 65:340-352.

49. Kennedy RD, Gorski JJ, Quinn JE, Stewart GE, James CR, Moore S, Mulligan K, Emberley ED, Lioe TF, Morrison PJ, Mullan PB, Reid G, Johnston PG, Watson PH and Harkin DP. BRCA1 and c-Myc associate to transcriptionally repress psoriasin, a DNA damage-inducible gene. Cancer Res. 2005; 65:10265-10272.

50. Friedman J, Hastie T and Tibshirani R. Regularization Paths for Generalized Linear Models via Coordinate Descent. Journal of statistical software. 2010; 33:1-22. 